\title{
Controlling Salmonella along the food chain in the European Union - progress over the last ten years
}

M Hugas (M.Hugas@efsa.europa.eu)1', P A Beloeil ${ }^{1}$

1. European Food Safety Agency, Parma, Italy

Citation style for this article:

Hugas M, Beloeil PA. Controlling Salmonella along the food chain in the European Union - progress over the last ten years. Euro Surveill. 2014;19(19):pii=20804.

Available online: http://www.eurosurveillance.org/ViewArticle.aspx?Articleld=20804

Salmonella has long been recognised as an important food-borne zoonotic pathogen of economic significance in animals and in humans. The main reservoir of Salmonella is the intestinal tract of a wide range of domestic and wild animals, which may result in contamination of a variety of foodstuffs of both animal and plant origin. This risk has been taken seriously by food business operators (FBO) and policy makers in the European Union (EU). The incremental implementation of an integrated legislative approach to monitor and control Salmonella along the food chain, from primary production to consumption, over the last ten years has thus brought about important progress, however, challenges remain as a paper by Kinross et al. about an ongoing EU-wide outbreak of $S$. Stanley in this issue demonstrates [1].

Animal and human surveillance of food-borne diseases in the European Union

In the EU, surveillance of food-borne salmonellosis in humans is mandatory [2, 3]. Food-borne outbreaks need to be thoroughly epidemiologically investigated [4]. Zoonoses and zoonotic agents, including Salmonella, are consistently monitored in food-producing animals and food thereof in EU countries [4]. Data on humans, animals and food are compiled and analysed jointly by the European Food Safety Agency (EFSA) and the European Centre for Disease Prevention and Control (ECDC) and presented annually in the EU Summary Report on trends and sources of zoonoses, zoonotic agents and foodborne outbreaks.

The 2012 report showed that, as in previous years, S. Enteritidis, S. Typhimurium and monophasic $S$. Typhimurium 1,4,[5],12:i:- were, by far, the serovars most frequently associated with human illness (Figure 1), followed by $S$. Infantis. S. Stanley was the causative pathogen in $0.8 \%$ and $1.4 \%$ of the human cases in 2011 and 2012, respectively [5]. Human S. Enteritidis cases are most commonly associated with the consumption of contaminated eggs and poultry meat, while $S$. Typhimurium cases are mostly associated with the consumption of contaminated pig, poultry and bovine meat [5].
Salmonella has been isolated from a wide range of foodstuff, but typically from various types of meat and meat products. In 2012, the highest proportions of Salmonella-positive single samples were reported for fresh broiler meat and turkey meat at an average level of $5.5 \%$, while in fresh pig and bovine meat, the proportions equalled $0.7 \%$ and $0.2 \%$, respectively, for the group of reporting countries [5].

National control programmes of Salmonella in poultry In animals, in particular in poultry, Salmonella causes mostly sub-clinical infections and the organism may easily spread between animals in a herd or flock without detection; animals can become intermittent or persistent healthy carriers. The prevalence of Salmonella in poultry populations is considered as the main risk factor for presence of Salmonella in table eggs and poultry meat. In order to control Salmonella in various production types of domestic fowls and turkeys, and to limit the risk of contamination of poultry products, national control and surveillance programmes (NCP) of Salmonella have been implemented in the countries in accordance with the EU legislation [6].

NCP targeting several Salmonella serovars deemed to be of particular public health significance were set up in selected poultry populations, such as breeding flocks of Gallus gallus, laying hens and broilers, as well as breeding and fattening turkeys based on the evidence that these populations have the highest risk of transmitting Salmonella. The target Salmonella serovars include S. Enteritidis, S. Typhimurium, S. Hadar, $S$. Infantis and $S$. Virchow in breeding flocks of Gallus gallus and $S$. Enteritidis and $S$. Typhimurium in the additional poultry populations covered by the programmes. S. Typhimurium also includes monophasic S. Typhimurium 1,4,[5],12:i:-.

NCP may vary to some extent between countries; nevertheless, they are based on the same principles and aims. NCP typically include systematic implementation of preventive measures of flock infection with Salmonella, thorough surveillance of the Salmonella status of flocks, and once a Salmonella infection is detected, implementation of control measures to 
Distribution of the 10 most common Salmonella serovars in humans in the European Union, $2012(\mathrm{~N}=82,409)$

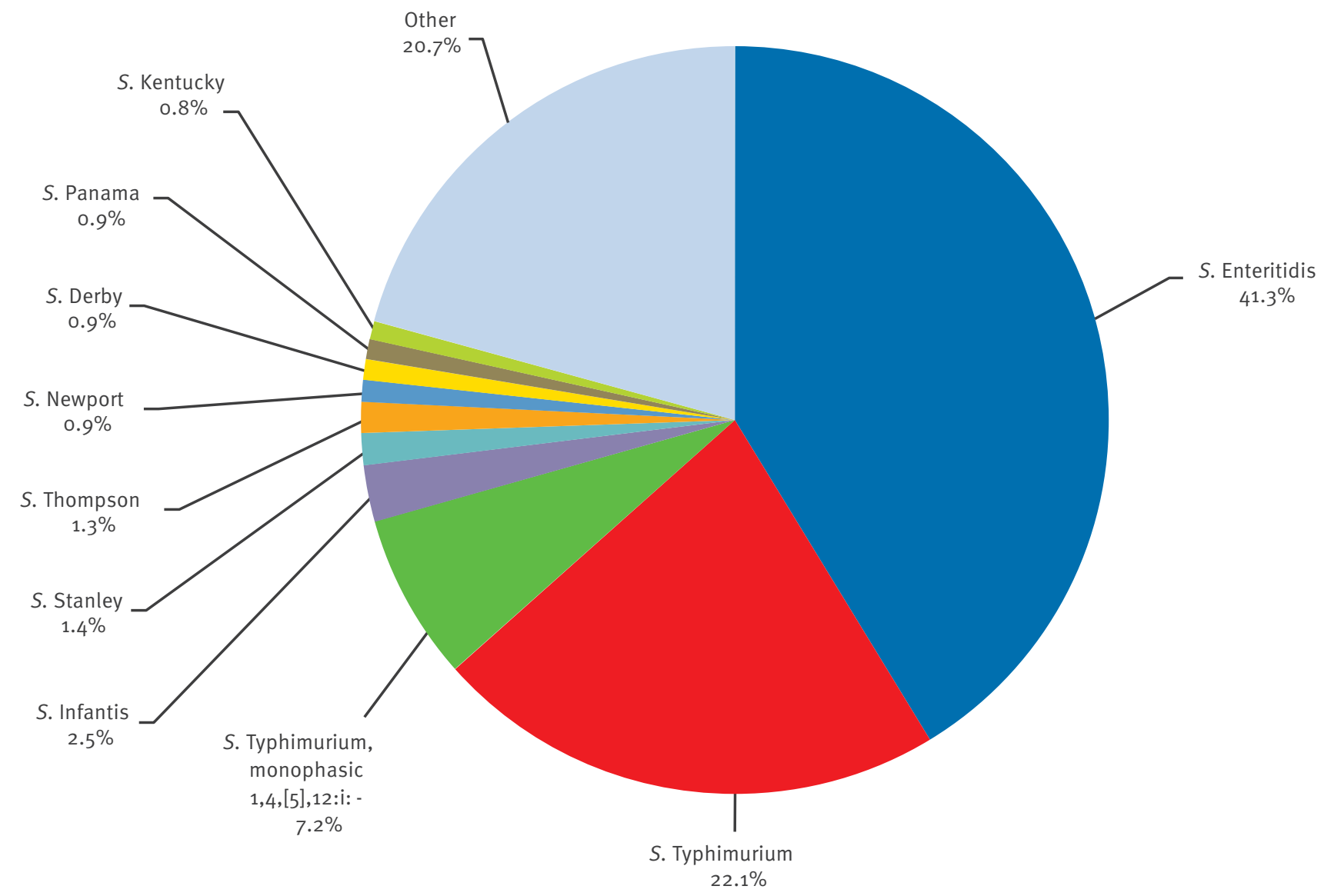

Source: [5]

prevent spread of infection. Poultry flocks are tested for the target Salmonella serovars at fixed stages of production at farms or hatcheries using harmonised sampling plans and standardised analytical methods.

With the exception of breeding flocks of Gallus gallus, EU Salmonella targets were set by the European Commission (EC) in consultation with EU Member States following EU-wide prevalence surveys [6]. The specific Salmonella control programmes and the reduction targets were progressively set up from 2005 onwards [7-14]. In the case of breeding and fattening turkey flocks, the mandatory NCPs for Salmonella came into effect on 1 January $2010[8,14,15]$ and were reconfirmed in 2013[16].

EFSA monitors whether EU targets for Salmonella prevalence reduction have been met by the countries and follows the progress made. Most countries met their Salmonella reduction targets for poultry in 2012, and the prevalence of the target Salmonella serovars is significantly declining or remaining stable in poultry populations at the EU level [5].

\section{Hygiene rules for Salmonella control in foodstuffs}

FBO are committed to general requirements on hygiene of foodstuffs, such as implementing procedures based on Hazard Analysis and Critical Control Points (HACCP) and good hygiene practices [17], and specific hygiene requirements with regard to unprocessed and processed products of animal origin, including poultry meat and meat products [18].

Furthermore, FBO should comply with specific food safety criteria for Salmonella in minced meat and meat preparations, in particular from poultry. These criteria define the acceptability of foodstuffs placed on the market. Complementary process hygiene criteria for Salmonella, notably on carcases of broilers and turkeys, set an indicative value above which corrective actions are required in order to maintain hygiene during processing [19].

Finally, official controls on products of animal origin intended for human consumption ensure that the legal framework for hygiene conditions is implemented correctly by $\mathrm{FBO}[20]$. 
Reported notification rates of zoonoses in confirmed ${ }^{\mathrm{a}, \mathrm{b}}$ human cases in the European Union, 2012

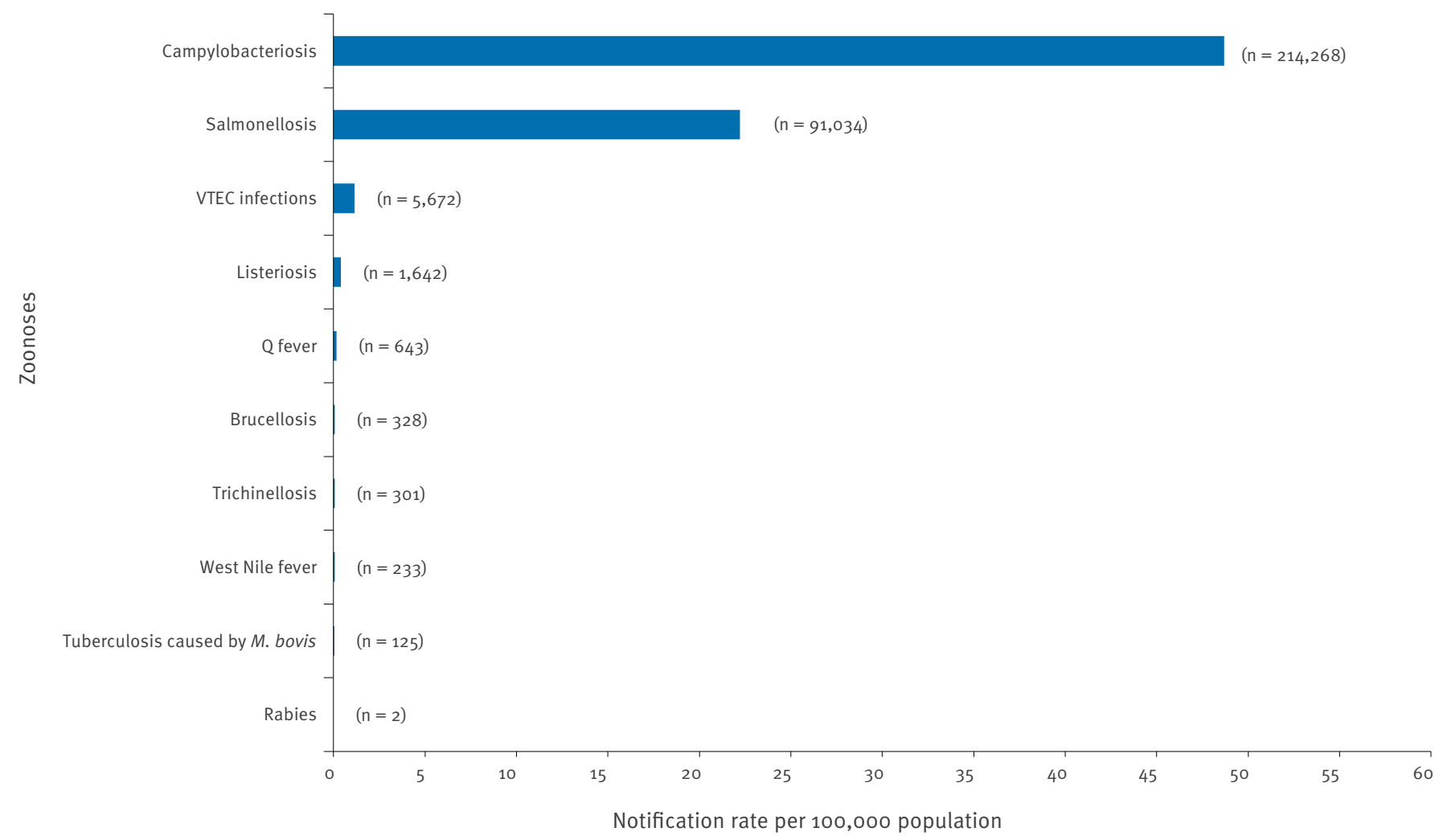

VTEC: verocytotoxin-producing E.Coli

Source: [5]

Note: Total number of confirmed cases is indicated in parenthesis at the end of each bar.

axception West Nile fever where total number of cases were used.

b Due to the restricted nature of the present report, the 2012 human notification rates for yersiniosis and echinococcosis were not produced but will be available in the "Annual Epidemiological Report 2014 - Reporting on 2012 surveillance data and 2013 epidemic intelligence data, ECDC 2014" (In preparation). The 2011 rates for these diseases were reported in the "The European Union Summary Report on Trends and Sources of Zoonoses, Zoonotic Agents and Food-borne Outbreaks in 2011; EFSA Journal 2013;11(4):3129".

\section{Success of Salmonella National Control Programmes}

In 2007, salmonellosis was the second most commonly reported zoonotic infection in the EU, with 151,995 human cases and a statistically significant decreasing trend in the notification rate in the EU over the past four years. [21]. The number of notified salmonellosis cases in humans in the EU continued to decrease in 2012, to 91,034 cases (Figure 2).This decline is part of the significant declining trend of $30 \%$ observed over the past five years. [5]. It is assumed that the observed reduction in salmonellosis cases in humans is mainly the result of successful Salmonella control programmes in fowl (Gallus gallus) populations particularly resulting in a lower occurrence of Salmonella in eggs, though other control measures might also have contributed to the reduction.

These results indicate that FBO and veterinary public health authorities have continued to invest in Salmonella control and that this work is yielding improvements even though challenges remain.
Challenge through possible prevalent serovars in poultry production sectors

Notwithstanding the positive developments, other Salmonella serovars, than the major targeted ones, may be occasionally implicated in food-borne outbreaks. In this issue of Eurosurveillance, Kinross et al. report about a cross-border outbreak of an unusual strain of serovar Salmonella Stanley that occurred in 2011-12 with more than 700 non-travel related human cases reported in 10 EU countries [1]. This number probably only represents the tip of the iceberg because additional cases might have not been captured by the surveillance systems in different countries. The investigations undertaken by affected countries, and subsequently coordinated by the European Commission ECDC and EFSA, suggested the turkey production chain as the source of the outbreak. More recently, further human cases of salmonellosis due to a $S$. Stanley strain exhibiting similar microbiological characteristics (i.e. resistance to ciprofloxacin) were detected and also linked to turkey meat in Austria in April 2014, 
suggesting that the outbreak may still be continuing with one or several similar sources $[1,22]$.

In March 2012, EFSA adopted a Scientific Opinion on an estimation of the public health impact of setting a new target for the reduction of Salmonella in turkeys [16]. It concluded that control measures in turkeys have contributed to a considerable reduction in the number of turkey-associated human salmonellosis cases compared with the situation in 2007. The target focusing on S. Enteritidis and S. Typhimurium, including monophasic $S$. Typhimurium, was therefore confirmed by the legislation for the period starting in 2013 onwards [14]. In addition, complete serotyping was required to inform on the diversity of serovars, other than the targeted ones, prevalent in flocks. Where necessary, targeted control of Salmonella serovars other than S. Enteritidis and $S$. Typhimurium in turkey should be guided by the prevalence and public health impact in each individual country. If sufficient information becomes available to reliably identify particular strains of public health significance, the inclusion of such strains as part of the EU-wide targets should be considered.

\section{References}

1. Kinross P, van Alphen L, Martinez Urtaza J, Struelens M, Takkinen J, Coulombier D, et al. Multidisciplinary investigation of a multicountry outbreak of Salmonella Stanley infections associated with turkey meat in the European Union, August 2011 to January 2013. Euro Surveill. 2014;19(19):pii=20801.

2. Decision No $2119 / 98 / E C$ of the European Parliament and of the Council of 24 September 1998 setting up a network for the epidemiological surveillance and control of communicable diseases in the Community. OJ L 268, 3.10.98, p. 1-6.

3. Commission Decision 2000/96/EC of 22 December 1999 on the communicable diseases to be progressively covered by the Community network under Decision No 2119/98/EC of the European Parliament and of the Council. OJ L 28, 3.2.2000, p. 50-53.

4. Directive $2003 / 99 / E C$ of the European Parliament and of the Council of 17 November 2003 on the monitoring of zoonoses and zoonotic agents, amending Council Decision 90/424/ EEC and repealing Council Directive 92/117/EEC. OJ L 325, 12.12.2003, p. 31-40.

5. European Food Safety Authority (EFSA), European Centre for Disease Prevention and Control (ECDC), 2014. The European Union Summary Report on Trends and Sources of Zoonoses, Zoonotic Agents and Food-borne Outbreaks in 2012. EFSA Journal 2014;12(2):3547, 312 pp. doi:10.2903/j.efsa.2014.3547.

6. Regulation (EC) No $2160 / 2003$ of the European Parliament and of the Council and Regulation of 17 November 2003 on the control of salmonella and other specified food-borne zoonotic agents. OJ L 325, 12.12.2003, p. 1-15.

7. Commission regulation (EC) No $1003 / 2005$ of 30 June 2005 implementing Regulation (EC) No $2160 / 2003$ as regards a Community target for the reduction of the prevalence of certain salmonella serotypes in breeding flocks of Gallus gallus and amending Regulation (EC) No 2160/2003. OJ L 170, 01.07.2005, p. $12-17$.

8. Commission regulation (EC) No 213/2009 of 18 March 2009 amending Regulation (EC) No 2160/2003 of the European Parliament and of the Council and Regulation (EC) No $1003 / 2005$ as regards the control and testing of Salmonella in breeding flocks of Gallus gallus and turkeys, OJ L 73 , 19.03.2009, p.5-11.

9. Commission Regulation (EC) No 200/2010 of 10 March 2010 implementing Regulation (EC) No 2160/2003 of the European Parliament and of the Council as regards a Union target for the reduction of the prevalence of Salmonella serotypes in adult breeding flocks of Gallus gallus. OJ L 61, 11.03.2010, p. 1-9.

10. Commission Regulation (EC) No $1168 / 2006$ of 31 July 2006 implementing Regulation (EC) No $2160 / 2003$ as regards a Community target for the reduction of the prevalence of certain salmonella serotypes in laying hens of Gallus gallus and amending Regulation (EC) No 1003/2005. OJ L 211, 01.08.2006. p. 4-8.

11. Commission Regulation (EC) No 517/2011 of 25 May 2011 implementing Regulation (EC) No 2160/2003 of the European Parliament and of the Council as regards a Union target for the reduction of the prevalence of certain Salmonella serotypes in laying hens of Gallus gallus and amending Regulation (EC) No $2160 / 2003$ and Commission Regulation (EU) No 200/2010. OJ L 138, 26.05.2011, p. 45-51.

12. Commission Regulation (EC) No 646/2007 implementing Regulation (EC) No $2160 / 2003$ of the European Parliament and of the Council as regards a Union target for the reduction of the prevalence of certain Salmonella enteritidis and Salmonella typhimurium in broilers and repealing Regulation (EC) No 1091/2005. OJ L 151, 13.06.2007, p. 21-25.

13. Commission Regulation (EC) No 200/2012 of 8 March 2012 concerning a Union target for the reduction of Salmonella enteritidis and Salmonella typhimurium in flocks of broilers, as provided for in Regulation (EC) No 2160/2003 of the European Parliament and of the Council. OJ L 71, 9.3.2012, p. 31-36.

14. Commission Regulation (EC) No 584/2008 implementing Regulation (EC) No 2160/2003 of the European Parliament and of the Council as regards a Union target for the reduction of the prevalence of certain Salmonella enteritidis and Salmonella typhimurium in turkeys. OJ L 162 21.06.2008, p. 3-8.

15. Commission regulation (EU) No 1190/2012 of 12 December 2012 concerning a Union target for the reduction of Salmonella Enteritidis and Salmonella Typhimurium in flocks of turkeys, as provided for in Regulation (EC) No 2160/2003 of the European Parliament and of the Council, OJ L 340, 13.12.2012, p. 29-34.

16. EFSA Panel on Biological Hazards (BIOHAZ); Scientific Opinion on an estimation of the public health impact of setting a new target for the reduction of Salmonella in turkeys. EFSA Journal 2012;10(4):2616. [89 pp.] doi:10.2903/j.efsa.2012.2616. Available online: www.efsa.europa.eu/efsajournal.

17. Regulation (EC) No 852/2004 of the European Parliament and of the Council of 29 April 2004 on the hygiene of foodstuffs, 0] L 139, 30.4.2004, p. 1.

18. Regulation (EC) No $853 / 2004$ of the European Parliament and of the Council of 29 April 2004 laying down specific hygiene rules for food of animal origin, OJ L 139, 30.4.2004, p. 55.

19. Regulation (EC) No $2073 / 2005$ of 15 November 2005 on microbiological criteria for foodstuffs, OJ L 338, 22.12.2005, p. 1.

20. Regulation (EC) No 854/2004 of the European Parliament and of the Council of 29 April 2004 laying down specific rules for the organisation of official controls on products of animal origin intended for human consumption. OJ L 139, 30.4.2004, p. 206.

21. Westrell T, Ciampa N, Boelaert F, Helwigh B, Korsgaard H, Chríel M, Ammon A, Mäkelä P. Zoonotic infections in Europe in 2007: a summary of the EFSA-ECDC annual report. Euro Surveill. 2009;14(3):pii=19100. Available online: http://www. eurosurveillance.org/ViewArticle.aspx?Articleld=19100

22. European Centre for Disease Prevention and Control (ECDC), European Food Safety Authority (EFSA). Multi-country outbreak of Salmonella Stanley infections - Third update, 8 May 2014. Stockholm and Parma: ECDC/EFSA; 2014. Available from: http://www.ecdc.europa.eu/en/publications/Publications/ salmonella-stanley-multi-country-outbreak-assessment-8May-2014.pdf 